\title{
Quality improvement in Respiratory Medicine: Designing and implementing a bronchoscopy checklist at Southend University Hospital
}

\author{
Muhunthan Thillai, Duncan Powrie
}

\begin{abstract}
There is currently no standardised procedure checklist for bronchoscopies in the United Kingdom. Furthermore, very few hospitals appear to routinely use any form of checklist for these procedures. Conversely, the World Health Organisation surgical checklist has become standard practice in most hospitals, our own included, for all surgical procedures. Based on our own needs, we decided to create a bronchoscopy checklist which could be used at our hospital with the primary emphasis on ensuring patient safety and improving clinical outcomes. We surveyed local surgical checklists as well as endoscopic checklists used in hospitals around the world. We then created an initial draft which was sent out to consultation to all Respiratory Physicians in our hospital. Comments were also taken from the endoscopy nursing staff. After a series of consultations, a checklist was trialled over the course of two weeks. Feedback from use in the endoscopy suite resulted in further modifications and a final version of the checklist came into use in May 2013. It is now used for all bronchoscopic procedures carried out by Respiratory Physicians in the hospital. An audit is planned for later this year to monitor uptake of the checklist and to determine if it has changed outcomes.
\end{abstract}

\section{Problem}

Bronchoscopies at our unit at Southend University Hospital are carried out by four consultants and one associate specialist who operate for three sessions a week between them. There are also up to three trainee doctors who may perform the procedure with assistance from a senior clinician. This results in up to eight different individuals and a maximum of 15 procedures each week. Additional bronchoscopies are carried out on the critical care unit as and when needed. All procedures are recorded by the bronchoscopist using a standard written proforma which incorporates the type and amount of local anaesthetic and sedation given, any abnormalities seen in the bronchial tree and the site of where samples are taken from. In addition, nursing staff have their own checklist for each patient including ensuring that the correct patient is identified and that wristband identification tags are used.

A number of verbal checks are made by the bronchoscopist but these are user dependent. They are not uniformly performed and vary in their level of assessment. These checks include inspection of the relevant radiology prior to the procedure, checking of anticoagulation status and determining which samples may need to be taken. Post procedure checks including measurement of oxygen saturations and heart rate are also made but again these are user dependent and there is no formal recording of these observations. Crucially, the communication between the different members of the team is not formalised which can potentially lead to delays and difficulties with patient management.

\section{Background}

In the 12 months leading up to May 2013, we carried out 269 bronchoscopies in our hospital and no major adverse events were reported. Over the past five years there have been a number of minor events but no reported major adverse events.

The British Thoracic Society guidelines for the use of flexible bronchoscopy is due to be updated later in 2013 but the current guidance confirms that flexible bronchoscopy is a relatively safe procedure (1). The largest study to date in the UK analysed retrospective data from 40,000 procedures and concluded that the mortality rate was $0.04 \%$ and the major complication rate was $0.12 \%$ (2). However, the complication rate is higher in patients at higher risk (e.g. those with co-existing cardiac or renal disease) and is also higher with specific procedures e.g. the overall mortality rate when taking transbronchial biopsies has been reported as being $0.1 \%$ with the rate of major complications as high as $6.8 \%$ (3). Major complications associated with the procedure include respiratory failure, cardiac dysrhythmias and bleeding whereas minor complications include vasovagal reactions and adverse effects of the local anaesthetic or sedation (4).

The World Health Organisation has developed a surgical checklist template which is adapted and in use in most hospitals in the United Kingdom (5). This simple list ensures good communication between members of the surgical team and has a series of pre- and postoperative checks. Variations have been adapted for use in nonsurgical procedures e.g. at Johns Hopkins the use of a checklist for inserting central lines has been shown to dramatically reduce the incidence of line associated complications such as line-associated infections and ventilator-associated pneumonia, thus improving patient safety and dramatically reducing cost (6). There is no such list available in widespread use for endoscopic procedures of any nature in the United Kingdom.

\section{Baseline Measurement}

A review of the notes from 20 patients having bronchoscopies between December 2012 and February 2013 revealed a wide 
variation in the standard of record keeping. In all cases, bronchoscopy forms were filled in appropriately and no major or minor complications appear to have occurred for any case. However, in only 3 patients, post procedure observations were recorded in the medical notes and in one case, a direct communication to the ward was recorded in order to hand over care to the team looking after the patient post procedure. In none of the 20 cases was a record made of acknowledgement of reviews of the CT scans, spirometry or blood clotting profile prior to the procedure. Although it is likely, based on current practice, that this was routinely checked, there is no written record of this having been carried out.

\section{Design}

The decision to create a bronchoscopy checklist was made after discussions with our consultants regarding the standardisation of pre and post-bronchoscopy checks. Although there had been no major complications in recent years, it was generally felt that better documentation and a more rigorous system of checks would be to our patients' benefit. A review of the literature confirmed that very few endoscopy checklists are routinely used by clinicians either in the United Kingdom or worldwide. This is stark contrast with the WHO surgical checklists which were devised less than a decade ago and are now in routine practice for operations across the world. The WHO surgical checklist was reviewed when creating a draft bronchoscopic checklist. Endoscopy checklists themselves are not in routine use worldwide but we assessed a number of these prior to writing our own draft e.g. the bronchoscopy checklist used in Vancouver, Canada (7).

Prior to the creation of our first draft, a detailed consultation period commenced which involved the four consultants and one associate specialist as well as the head nurse of our endoscopy unit and the two endoscopy nurses who had the greatest experience with bronchoscopy. A number of important objectives were agreed in this initial period. Firstly, we decided that the checklist should be confined to a single piece of paper and filed in the notes of all patients having the procedure. It should be filled in by the bronchoscopist and countersigned by a senior physician if the procedure was performed by a doctor in training. It was also decided that the checklist would have one section for checks prior to the procedure and one section for the post-procedure checks. It should be simple to use there should be no ambiguity about the checklist boxes. Finally, as with the WHO surgical checklist, it was decided that at the beginning of each bronchoscopy list there should be an opportunity for all members of the team to introduce themselves at the beginning of the list and voice any concerns that they may have before starting.

\section{Strategy}

PDSA Cycle 1

Through the use of the hospital email and intranet system, a virtual board of experts was created which consisted of all the Respiratory Physicians, Specialist Registrars and relevant endoscopy nurses at
Southend Hospital. The board was initially consulted on what they felt would be the most valuable additions to the checklist. These were then compiled into an adapted version of the standardised WHO Surgical Checklist. This was then made to concisely fit into one single sheet of A4 paper which would fit into the medical notes. This formed the first draft of the checklist.

\section{PDSA Cycle 2}

The first draft of the checklist was then sent to all board members and initial comments were assessed and changes to the checklist made where needed. These changes included the decision to have a specific section for infection control measures in cases of suspected tuberculosis, ensuring that nursing staff have labelled the sample pots correctly and deciding whether the clinician looking after the patient would need to be informed in cases of suspected lung cancer so that the patient is added for discussion at the next available lung cancer multidisciplinary meeting.

\section{PDSA Cycle 3}

After the amendments were made, the checklist was again circulated amongst the board to ask for minor amendments. These included a change in the size of the boxes to ensure that they were large enough to be ticked with ease and an explanation that that ticking a box meant that the point had been considered, even if it was not relevant e.g. ticking the box for 'clotting profile (if applicable)' would mean that the user had thought about this point, even if the patient was not on anticoagulants and had not therefore had a clotting profile checked as standard prior to the procedure.

\section{PDSA Cycle 4}

Up until this point the checklist had only existed in an electronic version. The latest draft was then printed onto paper and this was circulated to all board members. This allowed for final minor comments to be made which would only have been made on inspection of the actual checklist on paper e.g. it was felt that there was not enough space in between the tick boxes and this was addressed to make the checklist easier to use.

\section{PDSA Cycle 5}

The bronchoscopy checklist was then trialled for two weeks in the endoscopy department ensuring that all respiratory physicians and relevant endoscopy staff had the opportunity to use it. This ensured that comments made from a real world use of the checklist were incorporated into the next version of the checklist e.g. it was felt that the area for the patient identification sticker should be made larger and bolder to ensure that it was easily accessible to all members of staff.

\section{PDSA Cycle 6}

After taking on board these further comments, a final version of the checklist was produced. The official logo of the hospital was sought from the hospital communications department and this was added to the checklist. The decision made to use the checklist as a 
standard was then made at the monthly Respiratory Governance Meeting.

\section{Results}

Figure 1 shows the final version of the bronchoscopy checklist. This has been in circulation for two months and is now used routinely for all bronchoscopies in the department as well as those carried out by Respiratory Physicians on patients in intensive care. There have been no reported problems with it to date but a record will be kept of any difficulties encountered by either the medical team or the endoscopy nursing staff and this will be fed back to us at a later date. Any major issues will be collated and we will discuss them at a Respiratory Medicine Governance meeting 6 months after starting the list.

See supplementary file: ds1911.pdf - "Figure 1: Final version of the bronchoscopy checklist"

\section{Lessons and Limitations}

We have learnt a number of lessons from this project. Firstly, when implementing a new protocol it is important to get views from all members of the clinical team involved with the procedure. In this case we canvassed the view of our four consultants and one associate specialist as well as trainee doctors. It is also vital to get views from clinical staff who are from a non-medical background-in this case we took on board written comments by the head nurse in endoscopy as well as those from other nurses in the department. Finally, we learnt that use of the checklist in the real world is important as it acts as a 'post-deployment' assessment of the work. The implementation of the checklist over the two week pilot period allowed us to incorporate comments from users and discover unseen issues that were only uncovered once the checklist was in use.

There are however a number of limitations with this strategy. Firstly, the low incidence of mortality or major complications associated with bronchoscopies means that it will be difficult to tell initially whether our checklist has improved patient safety. However, it will allow for assessment of whether routine checks are carried out e.g. checking of the clotting profile and spirometry prior to bronchoscopy. As with the creation of the checklist, we will need to rely on qualitative reporting from the users and we have put in place a framework for this to begin. Additionally, we will need to continue to use the checklist on a regular basis in order to see if any further difficulties arise. Finally, it is important that we devise an accurate method of auditing use of the checklist to ensure that it is properly used by all bronchoscopists and that any difficulties which arise with it's use are properly documented and acted upon.

\section{Conclusion}

Bronchoscopies at Southend University Hospital are currently carried out by up to eight individuals i.e. four Respiratory Consultants, one Associate Specialist and three trainees. Until the development of a checklist there was no standardised method for pre and post-procedure checks. If such checks were carried out, they were done so on an individualised basis and no written record was kept. Despite having had no major adverse effects in the five years leading up the development of a checklist, it was widely felt that the introduction of a checklist would be beneficial to patients and ensure good clinical practice. The standardised WHO surgical checklist was adapted to our use and a number of specific changes were made after a series of consultations with all members of staff who use the checklist.

We feel that the creation and implementation of a bronchoscopy checklist is not simply about reducing the risks involved with the procedure but also in ensuring best practice for our patients. The checklist has been in regular use since May 2013 and feedback from both the users and the nursing staff in endoscopy indicate that it is a worthwhile measure to ensure a high level of safety for the procedures. The use of the checklist will be audited and these results collated with any difficulties encountered with the checklist. These will be fed back to our Respiratory Medicine Governance meeting and if needed, the checklist will be further modified.

\section{References}

1. British Thoracic Society Guidelines on Diagnostic Flexible Bronchoscopy. The British Thoracic Society Bronchoscopy Guideline Committee: a sub-Committee of the Standards of Care Committee of the British Thoracic Society - Thorax 2001. 56: (Suppl I); i1-i 21.

2. Simpson FG, Arnold AG, Purvis A, et al. Postal survey of bronchoscopic practice by physicians in the United Kingdom. Thorax 1986;41:311-7.

3. Pue C, Pacht E. Complications of fiberoptic bronchoscopy at a university hospital. Chest 1995;107:430-2.

4. Zavala DC. Complications following fiberoptic bronchoscopy. Chest 1978;73:783-5.

5. World Health Organisation Surgical Checklist version 2009: http://www.safesurg.org/uploads/1/0/9/0/1090835/surgical_s afety_checklist_production.pdf

6. Kellie SP, Scott MJ, Cavallazzi R, Wiemken TL, Goss L, Parker D, Saad M. Procedural and Educational Interventions to Reduce Ventilator-Associated Pneumonia Rate and Central Line-Associated Blood Stream Infection Rate. J Intensive Care Med. 2012 Nov 15.

7. Vancouver bronhcosocpy checklist: http://bcpsqc.ca/documents/2012/09/procedural safety che cklist_bronchoscopy.pdf

\section{Declaration of interests}

None declared

\section{Acknowledgements}

We would like to thank all who were involved with designing the checklist, specifically: Masood Ali, Sohail Ansari, Peter Bailey, Philip Branch, Farag Eltaie, Vera Gummer, Alicia Howe, Ganes Lingam and Abigail Moore. 